\title{
Initial Analysis of Lipid Metabolomic Profile Reveals Differential Expression Features in Myeloid Malignancies
}

\author{
Adriana Ramos de Oliveira1, Ismael Dale Cotrim Guerreiro Da Silva², Edson G. Lo Turco ${ }^{2}$, \\ Helio Alves Martins Júnior', Maria de Lourdes L. Ferrari Chauffaille ${ }^{1^{*}}$ \\ ${ }^{1}$ Department of Clinical and Experimental Oncology, Federal University of São Paulo, São Paulo, Brazil \\ ${ }^{2}$ Gynecologic Department, Federal University of São Paulo, São Paulo, Brazil \\ Email: drikabiomed@yahoo.com.br, "chauffaille@unifesp.br
}

Received 12 November 2015; accepted 21 December 2015; published 25 December 2015

Copyright (C) 2015 by authors and Scientific Research Publishing Inc.

This work is licensed under the Creative Commons Attribution International License (CC BY). http://creativecommons.org/licenses/by/4.0/

(c) (i) Open Access

\section{Abstract}

The purpose of this preliminary study was to determine the comparative lipid profile of blood plasma samples of healthy individuals and patients with Myeloproliferative Neoplasms. Methods: Untargeted Shotgun MS/MS Analysis was performed to evaluate plasma samples from 153 participants, being 90 of the Control Group, 43 Myeloproliferative Neoplasms (MPN), 11 Myelodysplastic Syndromes (MDS) and 9 Acute Myeloid Leukemias (AML). Lipids were extracted from plasma using the Bligh-Dyer protocol. Data were acquired using the AB-Sciex Analyst TF, processed using the AB-Sciex LipidView ${ }^{\mathrm{TM}}$ and the web-based analytical pipeline MetaboAnalyst 2.0

(www.metaboanalyst.ca). Results: Untargeted analysis identified in negative and positive-modes a total of 658 features at $2 \mathrm{ppm}$ resolution. PCA and PLS-DA analysis revealed clear discrimination among groups, in particular for AML patients. Main lipid groups differentially expressed were: Monoacylglycerols (MAG), Glucosylceramide E (GlcdE), Ethyl Esters (EE), Lysophosphatidic acid (LPA), Sulfoquinovosil diacylglycerols (SQDG), Monoglycerols (MG), Methyl Ethanolamines (ME), Lysophosphatidylcholines (LPC), Dimethyl Phosfatidyletanolamines (DMPE), Monometylphosphatidiletanolamines (MMPE), Ceramide-1-phosphate (CerP), Glicerophosphoglycerols (GP), Lysomonomethyl-Phosphatidylethanolamines (LMMPE), Phosphatidic Acids (PA), Ergosterols (ERG), Glycerophosphoserine (PS), Diacylglycerols (DAG), Hexocylceramides (HexCer) and Lanosterol (Lan). ROC Curve Analysis revealed Total LMMPE as the strongest discriminating marker between Controls from Patients. In addition, these lipids were also able to differentiate MDS and AML from NPM. Conclusions: The Myeloproliferative Neoplasms from the point of view of global plasma lipidomics are accompanied by several modifications. In particular, the Lysomonomethyl-Phosphatidylethanolamines (LMMPE) seems to play important differentiating roles among them.

\footnotetext{
${ }^{*}$ Corresponding author.
} 


\section{Keywords}

\section{Lipids, Lipidomics, Myeloproliferative Neoplasms, Mass Spectrometry}

\section{Introduction}

Myeloid Neoplasms are clonal diseases of hematopoietic stem cells or progenitor cells that can be present at bone marrow and/or peripheral blood. Genetic or epigenetic alterations lead to functional disturbance of those cells, as well as harmed capacity of self-renewal, proliferation and differentiation.

This group of diseases was classified by World Health Organization (WHO) in five main types: Acute Myeloid Leukemias (AML), Myelodysplastic Syndromes (MDS), Myeloproliferative Neoplasms (MPN), Myeloproliferative/Myelodysplastic (MPN/MDS) and Myeloid Neoplasies associated with eosinophilia and abnormalities of growth factor receivers-PDGFRA, PDGFRB or FGFR1 genes [1]. This classification is grounded on characteristic morphology, cytochemistry, imunophenotyping, genetics and clinic. Approximately 35.000 cases of Leukemia are diagnosed in the USA each year; in other words, it represents a rate of 8.5 cases to 100.000 inhabitants and such rate has been steady within the last 30 years [2].

The lipidic metabolism in cancer, which a short time ago was predominantly studied at genetic level, has been gaining additional interest. From four types of biological molecules that form the human body, in other words nucleic acids, amino acids, carbohydrates and lipids, the latter stands out among many cellular metabolites, as different chemical entities. Although it is not well-defined, there are estimates that there are between 10.000 and 100.000 different molecular species in lipidic sphere [3]-[5]. Its functions were initially related to composition of biological membranes and storage of energy, but recently, those molecules have been analyzed under the aspect of different functions and regulatory and signaling effect [6].

The widest study of lipidic molecules, aiming at its global characterization and interaction with proteins and signalization ways, including genetic regulation and its functions related to homeostasis, was defined as lipodomic and shows up as an emerging field in studying different pathologies [6]. Metabolites of sphingolipids have been referenced as important survival modulators cellular growth, migration and angiogenesis, in addition to an important participation in cancer progression [7].

Throughout the last two decades, the mass spectrometry (MS) has emerged as the main method used in lipidomics analysis, which enables the structural characterization and quantification of complex lipids and its metabolites [8]. The analysis by such platform consists in generating ions grounded in compounds (either organic or inorganic ones) through appropriate ionization method. Subsequently, the ions are separated through their relationship mass-charge $(\mathrm{m} / \mathrm{z})$, in an analyzer of masses and qualitatively and/or quantitatively detected through a detector that "counts" ions.

The ionization type most often used is mass spectrometry through electrospray ionization (ESI), which has been one of most popular and powerful technologies for quantitative analysis of lipidic species. Nowadays, two main analytical approaches from ESI have been applied: Liquid chromatography coupled to spectrometer of masses (LC-MS) and direct infusion [9]. The analysis through MS as direct infusion of solution containing analytes of interest has been called "shotgun lipidomics" and was the technique used in this work.

The blood plasma can capture proteins and metabolites released from all organs and tissues, both healthy and sick ones [10] [11]. Having in view the potential of lipidomic technological platform and publications of a number of researchers in some pathologies, we consider the innovative use of such approach to study possible modifications in plasmatic metabolomic lipidic profile in myeloid neoplasms, with the purpose of identifying potential biomarkers and understanding of participation of lipids in biological phenomena resulting from this condition.

\section{Material and Method}

\subsection{Study Drawing and Samples Obtainment}

It was conducted a lipidic profile study in samples of patients being followed on ambulatory of Hematology of UNIFESP-EPM and admitted into ward of Sao Paulo Hospital, including both those who already have a defined 
diagnosis and those that were admitted throughout the study. It was not considered the diagnosis or therapeutic moment, only the presence of disease. The samples were obtained by vein punction and it was collected approximately $5 \mathrm{~mL}$ of peripheral blood in a pipe with ethylenediamine tetraacetic acid (EDTA) anticoagulant.

The control group was constituted by volunteer donators of blood bank of Sao Paulo Hospital, elderly people of group of elderlies of ambulatory of Geriatrics subject of UNIFESP-EPM and elderly people from Cohabitation Nucleous of Elderly People (NCI) of Vila Mariana.

It was analyzed 153 samples, being 90 of Control group, 43 Myeloproliferative Neoplasms (MPN), 11 Myelodysplastic Syndromes (MDS) and 9 Myeloid Leukemias (AML). The samples were obtained from a total of 90 volunteer without history of neoplasia (46 women and 44 men with average age of 54.2 years old, range of 30 89 years); 43 samples of patients with Chronicle Myeloproliferative Neoplasms-16 Polycythemia Vera (PV), 10 Essential Thrombocythemia (ET) and 17 Myelofibrosis (MF) (20 women and 23 men with age average of 66.1 years old, range of 31 - 90 years); 11 samples of patients with Myelodysplastic Syndromes (8 women and 3 men with average age of 73.9 years, range of 39 - 93 years) and 9 samples of patients with Acute Myeloid Leukemia ( 4 women and 5 men with age average of 54.7 years, range of 31 - 79 years). It was included in the study patients with diagnosis of AML, both primary and secondary ones, MPN and MDS, classified according to WHO criteria and to which it was possible to obtain enough samples for the study. It was excluded patients who did not agree with signing the free and grounded consent document, those on which it was not possible, for any reason, to make the collection of sample for analysis or who did not fit into WHO classification as AML, both primary and secondary ones, MPN and MDS.

\subsection{Patients}

The work was approved by Research Ethics Committee of Federal University of Sao Paulo, under protocol 66344 (CAAE 05109812.0.0000.5505) and all procedures were carried out after signature of Free and Grounded Consent Document by control patients and individuals.

\subsection{Preparation of Sample to Analyze Lipids}

After collecting the total blood in EDTA pipe, the samples were immediately centrifuged to $2590 \mathrm{rpm}$ (800 g), for 10 minutes. The blood plasma was separated in micro pipe Eppendorf of $2 \mathrm{~mL}$ and frozen at $-80^{\circ} \mathrm{C}$ for subsequent extraction of lipids. The Phosphocholine Standard lipid D-9 was diluted in a concentration of $5 \mu \mathrm{g} / \mathrm{mL}$ from a store solution of concentration $5 \mathrm{mg} / \mathrm{mL}$.

\subsection{Lipids Extraction Procedure}

Each sample was submitted to protocol of lipids extraction of Bligh-Dyer type (Bligh and Dyer, 1959). It was added $100 \mu \mathrm{L}$ of plasma sample in an Eppendorf micro pipe immediately after defrosting. After that, it was added $250 \mu \mathrm{L}$ of chloroform, $500 \mu \mathrm{L}$ of methanol and $100 \mu \mathrm{L}$ of milli-Q water. It was added to each sample 0,1 $\mu \mathrm{L}$ of Phosphocholine Standard D-9. Then, the mix was agitated for 30 seconds, using a vortex enabling more contact of lipids of samples with solvents. After agitation, it was added to the pipe $250 \mu \mathrm{L}$ of chloroform and $200 \mu \mathrm{L}$ of milli-Q water. Subsequently, the mix was centrifuged on $1000 \mathrm{rpm}$, for 5 minutes, at room temperature. The phase containing lips (apolar phase) was slowly separated in another micro pipe, using a Pasteur pipette of $200 \mu \mathrm{L}$ to evaporate the chloroform, in room temperature, for subsequent storage at $-80^{\circ} \mathrm{C}$. To analyze mass spectrometry of ESI, it was added to each pipe of $400 \mu \mathrm{L}$ a solution of chloroform/methanol 1:1.

\subsection{Analysis by Masses Spectrometry of Electrospray Type}

In such study, the analisis of lipidomics were made in a hybrid masses spectrometer quadrupolo/tempo-de-vôo TripleTOF $^{\circledR} 5600$ (AB SCIEX, Concord, Canada) of high resolution and mass accuracy. The compounds were analyzed with ionization by electrospray in positive and negative modes, employing ionization source DuoSpray $^{\circledR}$.

The ionization source DuoSpray was used with desolvation temperature of $250^{\circ} \mathrm{C}$ and ionization voltage of 5 $\mathrm{kV}$ and $-4,5 \mathrm{kV}$, for positive and negative modes, respectively. Ultra pure air was used as nebulizing gas (GS1) and drying gas (GS2), both with pressure of 20 psi. Nitrogen was used as a desolvation curtain gas on pressure of 15 psi and as collision gas in collision cell (Q2). 
The analysis were made in mode scanning mode MS with dependent acquisition (IDA) of MS/MS scanning. The experiments of MS scanning (TOFMS) were conducted in interval from $\mathrm{m} / \mathrm{z} 200$ to 1200 with signal accumulation time of $250 \mathrm{~ms}$. The experiments depending on product ions scanning (MS/MS) were set for 20 most abundant signals in MS spectrums, with scanning from $\mathrm{m} / \mathrm{z} 100$ to 1500 and signal accumulation time of $30 \mathrm{~ms}$. It was employed an exclusion time of precursor ions of $42.5 \mathrm{~ms}$ for dependent acquisition experiments. The masses spectrometer was calibrated for a commercial mix of known compounds in order to keep the error of masses equal or inferior to $2 \mathrm{ppm}$.

The samples were analyzed by flow injection (FIA), under flow of mobile phase of $50 \mu \mathrm{L} \cdot \mathrm{min}^{-1}$ and total time of run of 3.5 minutes. The mobile phase was constituted of methanol/water $(95 / 05, \mathrm{v} / \mathrm{v})$ with $5 \mathrm{mmol} \cdot \mathrm{min}^{-1}$ of ammonium acetate. A final volume of $40 \mu \mathrm{L}$ of sample was injected in both ionization methods (positive and negative one). The isopropanol injections ( $50 \mu \mathrm{L}$ ) were interpolated with samples to clean the injection system and piping of HPLC. The acquisition and search of lipids data were carried out with programs Analyst TF (version 1.5.2) and LipidView (version 1.2).

\section{Outcomes}

\subsection{Data Processing}

From the acquisition of the spectra profiles presented by each group in the analysis by ESI-MS, differentiated by the relative intensities of the ions, started the statistical analysis. Data processing was performed in programs MetaboAnalyst 2.0 (http://www.metaboanalyst.ca) [12] and ROCCET: ROC Curve Explorer \& Tester (http://www.roccet.ca/ROCCET/). MetaboAnalyst is a web-based metabolomic data processing tool which it accepts a variety of input data (NMR peak lists, binned spectra, MS peak lists, compound/concentration data) in a wide variety of formats. It also offers a number of options for metabolomic data processing, data normalization, multivariate statistical analysis, graphing, metabolite identification and pathway mapping. This software supports such techniques as: fold change analysis, t-tests, PCA, PLS-DA, hierarchical clustering and a number of more sophisticated statistical or machine learning methods. To evaluate the statistical significance of each model, the ROC calculations included: Monte-Carlo 100-fold Cross Validation (MCCV), Bootstrap 95\% confidence intervals for the Desired model specificity, Accuracy and false discovery rates (FDR) and 1000-fold permutation tests.

\subsection{Untargeted Analysis}

Untargeted analysis identified in negative and positive-modes a total of 658 features at $2 \mathrm{ppm}$ resolution. In order to differential circulating lipids, a parameter VIP (Variable Importance in the Projection) was utilized to show the variable importance in the discriminant analysis. PCA and PLS-DA analysis (Figure 1(a) and Figure 1(b)) revealed clear discrimination among groups, in particular for AML patients. Main lipid groups differentially expressed were: Monoacylglycerols (MAG), Glucosylceramide E (GlcdE), Ethyl Esters (EE), Lysophosphatidic acid (LPA), Sulfoquinovosil diacylglycerols (SQDG), Monoglycerols (MG), Methyl Ethanolamines (ME), Lysophosphatidylcholines (LPC), Dimethyl Phosfatidyletanilamines (DMPE), Monometylphosphatidiletanolamines (MMPE), Ceramide-1-phosphate (CerP), Glicerophosphoglycerols (GP), Lysomonomethyl-Phosphatidylethanolamines (LMMPE), Phosphatidic Acids (PA), Ergosterols (ERG), Glycerophosphoserine (PS), Diacylglycerols (DAG), Hexocylceramides (HexCer) and Lanosterol (Lan). Analyzing each of the major lipid groups by ANOVA and PostHoc, found possible to find differences in expression in the studied phenotypes (Table 1). P-value, $-\log 10(\mathrm{p})$, and FDR are addiction statistics confirming results.

The Myeloproliferative Neoplasms the point of view of global plasma Lipidomic analyzes are accompanied by the some changes on lipid Glucosylceramide E groups (GlcdE) (pValue $=6.1194 \mathrm{E}-6-\log 10(\mathrm{p})=5.2133$ FDR $=6.4754 \mathrm{E}-5$ (Figure 2), Lysophosphatidic Acid (LPA) (pValue $=9.4861 \mathrm{E} 9-\log 10(\mathrm{p})=8.0229 \mathrm{FDR}=$ 4.1739E-7) (Figure 3), Sulfoquinovosyl Diacylglycerol (SQDG) $(\mathrm{pValue}=4.2158 \mathrm{E}-5-\log 10(\mathrm{p})=4.3751$ FDR $=2.7824 \mathrm{E}-4)($ Figure 4$)$, Ethyl Ester $(\mathrm{EE})(\mathrm{pValue}=4.5211 \mathrm{E}-9-\log 10(\mathrm{p})=8.3448 \mathrm{FDR}=2.9839 \mathrm{E}-7)$ (Figure 5) and Monoacylglycerol (MAG) (pValue $=1.1907 \mathrm{E}-8-\log 10(\mathrm{p})=7.9242 \mathrm{FDR}=1.8009 \mathrm{E}-7$ (Figure 6) (ANOVA and Post-Hoc) witch present different expressions showing greater concentration in MDS and AML groups and lower for the MPN and control groups. This fact brings us the curious and initial impression that these groups could be related to chronic and acute character of the studied phenotypes. 


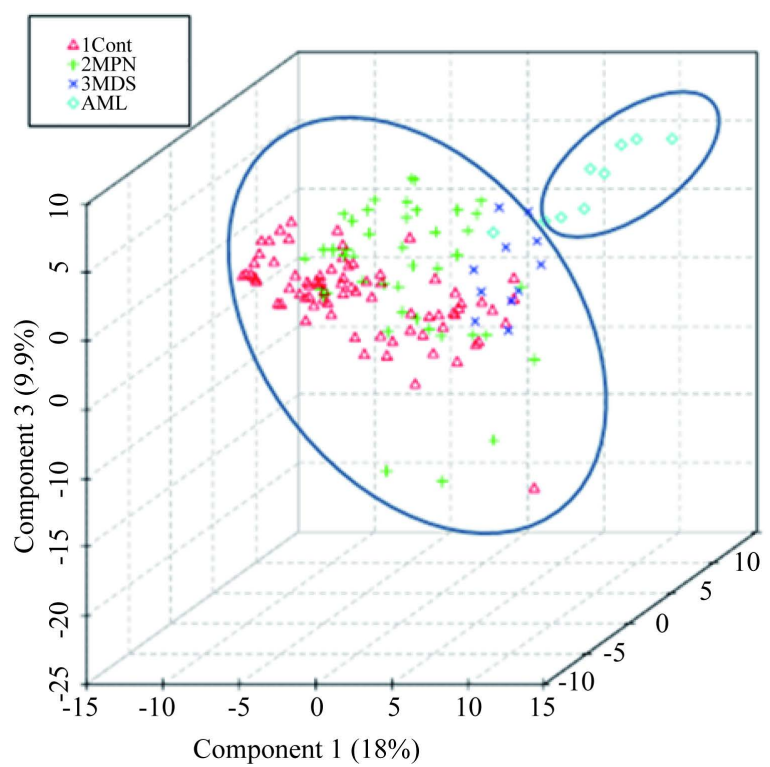

(a)

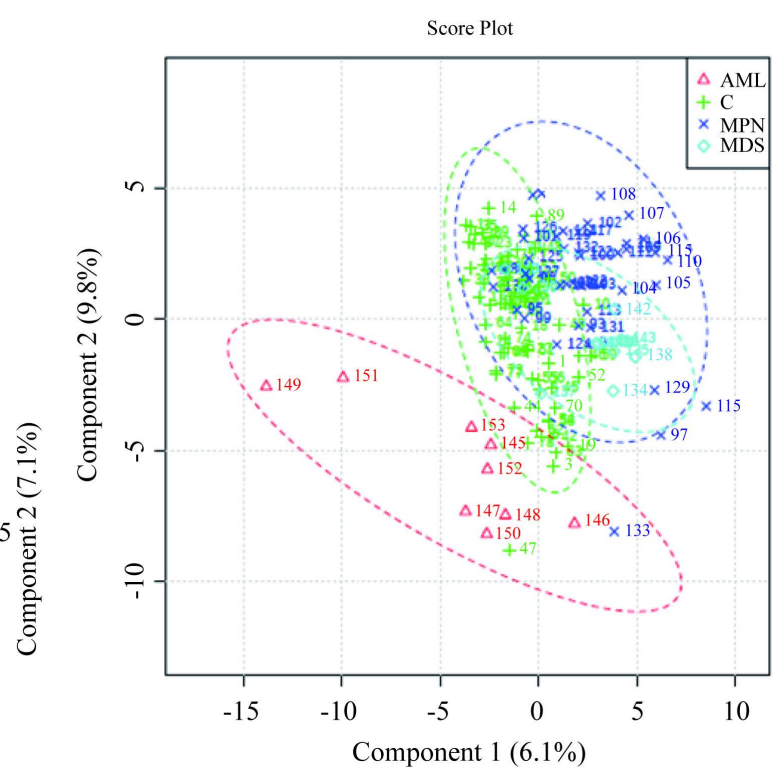

(b)

Figure 1. PCA and PLS-DA analysis. (a) show an "unsupervised" kind the variance between groups, considering the components 1 and 2, cluster individuals without regard to the division of the groups; (b) show the scores of the samples individually, considering the groups.

Table 1. Features identified by one-way ANOVA and post-hoc analysis.

\begin{tabular}{|c|c|c|c|c|}
\hline SPECTRA BINS & P-VALUE & $-\mathbf{L O G 1 0}(p)$ & FDR & FISCHER'S LSD \\
\hline Total LPA & $1.8311 \mathrm{E}-11$ & 10.737 & $4.7608 \mathrm{E}-10$ & MDS > C; AML > C; MDS > MPN; AML > MPN \\
\hline Total MAG/Total DAG & $6.5597 \mathrm{E}-11$ & 10.183 & $8.5276 \mathrm{E}-10$ & MDS > C; AML > C; MDS > MPN; AML > MPN \\
\hline Total EE & $2.1338 \mathrm{E}-10$ & 9.6708 & $1.8493 \mathrm{E}-9$ & AML > C; MDS > NPM; AML > MPN; AML > MDS \\
\hline Total MAG & 4.9119E-9 & 8.3088 & 3.1927E-8 & $\mathrm{C}>\mathrm{MPN}$; MDS $>\mathrm{C}$; AML $>\mathrm{C}$; MDS $>$ MPN; AML $>$ MPN \\
\hline Total LPA/Total PA & $3.8537 \mathrm{E}-8$ & 7.4141 & $2.0039 \mathrm{E}-7$ & MDS > C; AML > C; MDS - MPN; AML > MPN \\
\hline Total GlcdE & $7.7401 \mathrm{E}-8$ & 7.1113 & $3.354 \mathrm{E}-7$ & MPN > C; MDS > C; AML > C; MDS > MPN; AML > MPN \\
\hline Total Lan & $1.5867 \mathrm{E}-7$ & 6.7995 & $5.8934 \mathrm{E}-7$ & $\mathrm{C}>\mathrm{MPN}$; $\mathrm{C}>\mathrm{MDS}$; $\mathrm{C}>\mathrm{AML}$; MPN > MDS; MPN > AML \\
\hline Total HexCer & $9.299 \mathrm{E}-7$ & 6.0316 & $3.0222 \mathrm{E}-6$ & $\mathrm{C}>\mathrm{MPN}$; C > MPN; C > AML; MPN > MDS; MPN > AML \\
\hline Total Lipids & $7.0977 \mathrm{E}-6$ & 5.1489 & $2.0505 \mathrm{E}-5$ & $\mathrm{C}>$ MDS; C > AML; MPN > MDS; MPN > AML \\
\hline Total SQDG & $5.5002 \mathrm{E}-5$ & 4.2596 & $1.4301 \mathrm{E}-4$ & AML $>$ C; AML > MPN; AML > MDS \\
\hline Total LMMPE & $2.1591 \mathrm{E}-4$ & 3.6657 & $5.1033 \mathrm{E}-4$ & MPN > C; C > MDS; C > AML; MPN > MDS; MPN > AML \\
\hline Total Erg & $3.7084 \mathrm{E}-4$ & 3.4308 & $8.0349 \mathrm{E}-4$ & $\mathrm{C}>\mathrm{MPN} ; \mathrm{C}-\mathrm{MDS}$ \\
\hline Total ME & $5.5246 \mathrm{E}-4$ & 3.2577 & 0.0011049 & $\mathrm{MPN}>\mathrm{C}$; MDS $>\mathrm{C}$ \\
\hline PG 28:2 & 0.003713 & 2.4303 & 0.0068956 & $\mathrm{C}>\mathrm{MPN}$ \\
\hline Total DAG & 0.0052228 & 2.2821 & 0.0090529 & $\mathrm{C}>\mathrm{MPN}$; $\mathrm{C}>\mathrm{MDS} ; \mathrm{C}>\mathrm{AML}$ \\
\hline Total PA & 0.013743 & 1.8619 & 0.022332 & C - MDS \\
\hline PS $36: 5$ & 0.01547 & 1.8105 & 0.023659 & $\mathrm{C}>\mathrm{MDS} ; \mathrm{C}>\mathrm{AML}$ \\
\hline Total CerP & 0.017974 & 1.7454 & 0.025963 & MPN > C; MPN > MDS; MPN > AML \\
\hline
\end{tabular}


Total GlcdE

Original Conc.

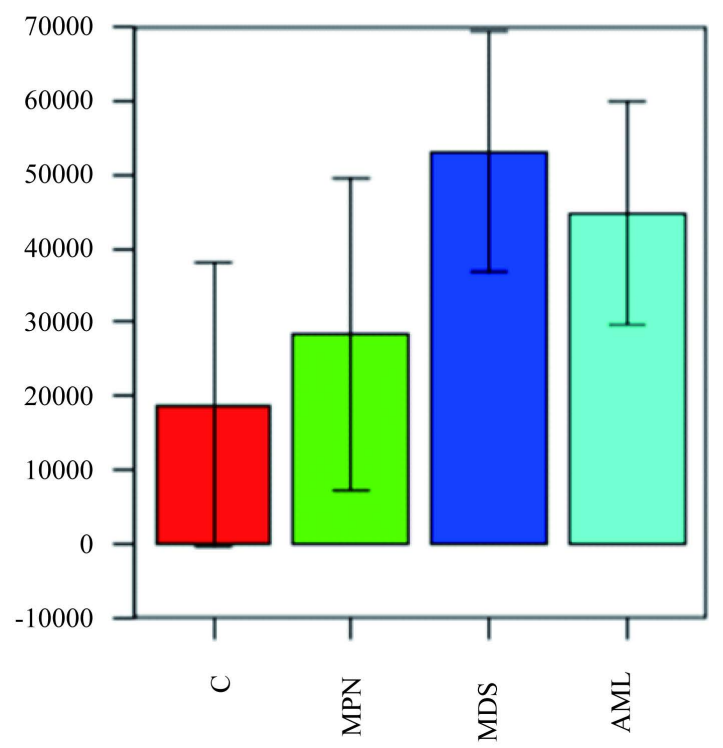

Normalized Conc.

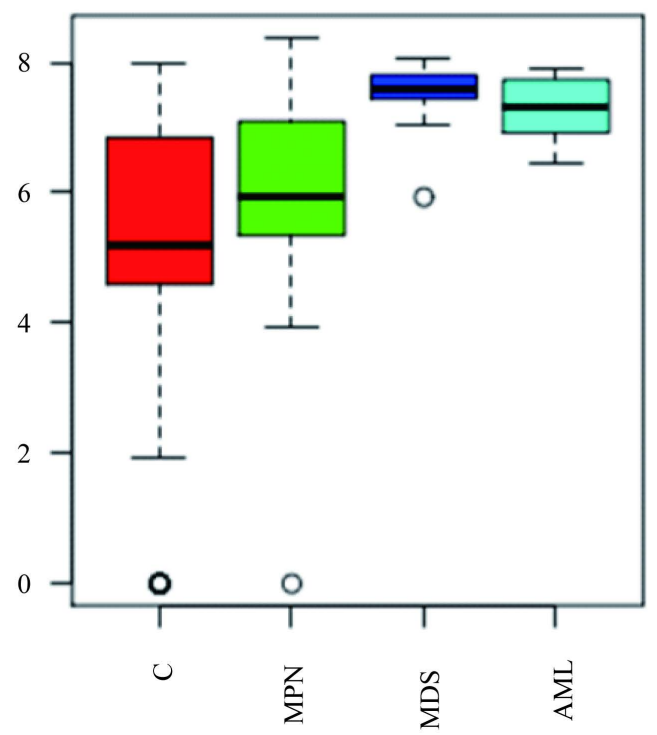

Figure 2. Total GlcdE is detected at different concentrations them control groups, MPN, MDS and AML. pValue $=6.1194 \mathrm{E}$ $-\log 10-6(\mathrm{p})=5.2133 ; \mathrm{FDR}=6.4754 \mathrm{E}-5$; MPN—cont; MDS—cont; AML—cont (ANOVA and Post-Hoc). C = Controls (n = 90); MPN = Myeloproliferative neoplasms ( $n=43)$; MDS = Myelodysplastic syndromes $(n=11)$; AML = Acute myeloid leukemia $(n=9)$.

\section{Total SQDG}

Original Conc.

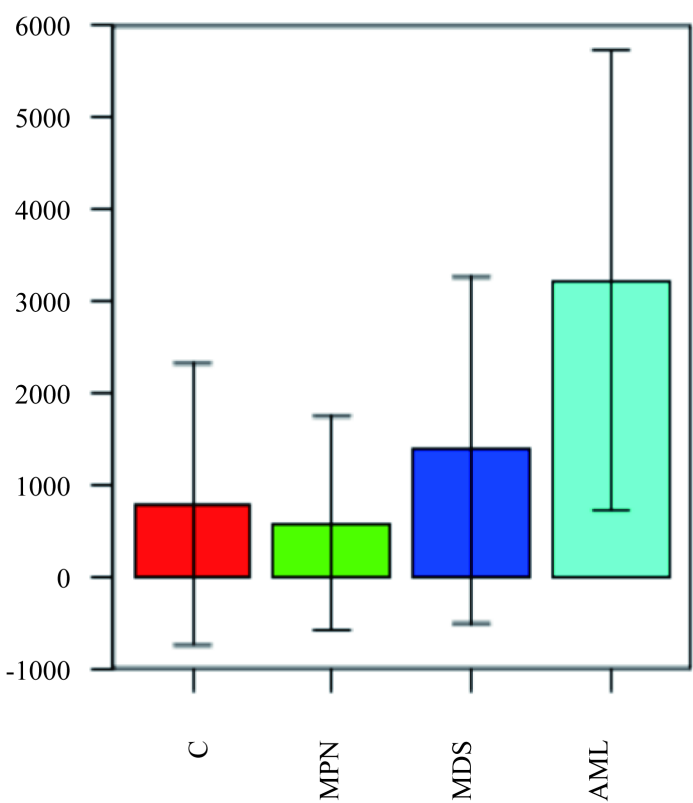

Normalized Conc.

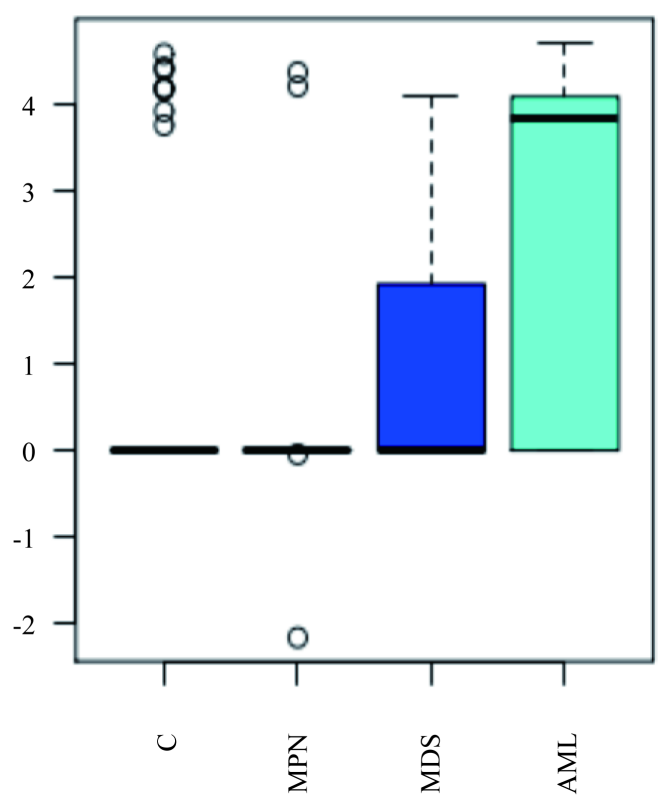

Figure 3. Total SQDG is detected at different concentrations them control groups, MPN, MDS and AML. pValue = 4.2158E-5 - $\log 10(\mathrm{p})=4.3751 ; \mathrm{FDR}=2.7824 \mathrm{E}-4$; AML-cont; AML-MPN; AML-MDS (ANOVA and Post-Hoc). C = Controls $(n=90)$; MPN = Myeloproliferative neoplasms $(n=43)$; MDS = Myelodysplastic syndromes $(n=11) ;$ AML = Acute myeloid leukemia $(n=9)$. 
Total LPA

Original Conc.

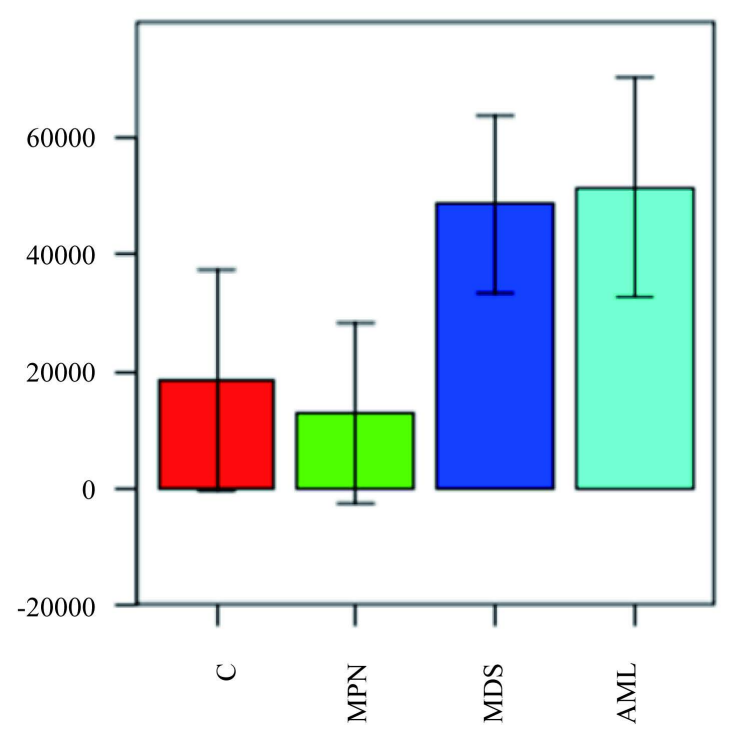

Normalized Conc.

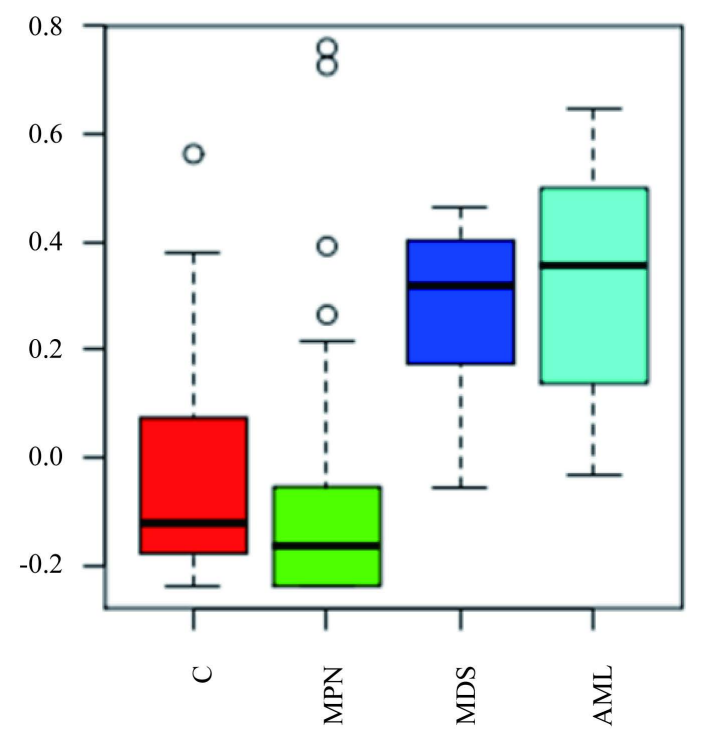

Figure 4. Total LPA is detected at concentrations them different control groups, MPN, MDS and AML. pValue = 9.4861E-9 - $\log 10(\mathrm{p})=8.0229 ;$ FDR = 4.1739E-7; MDS-cont; AM-cont; MDS-MPN; AML-MPN (ANOVA and Post-Hoc). $C=$ Controls $(n=90)$; MPN = Myeloproliferative neoplasms $(n=43)$; MDS = Myelodysplastic syndromes $(n=$ $11) ;$ AML = Acute myeloid leukemia $(n=9)$.

Total EE

Original Conc.

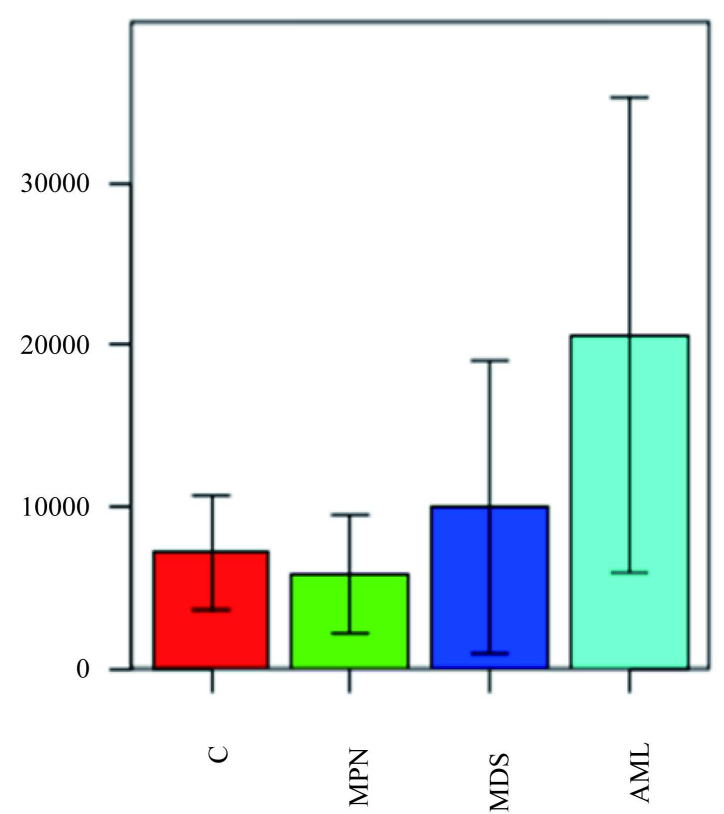

Normalized Conc.

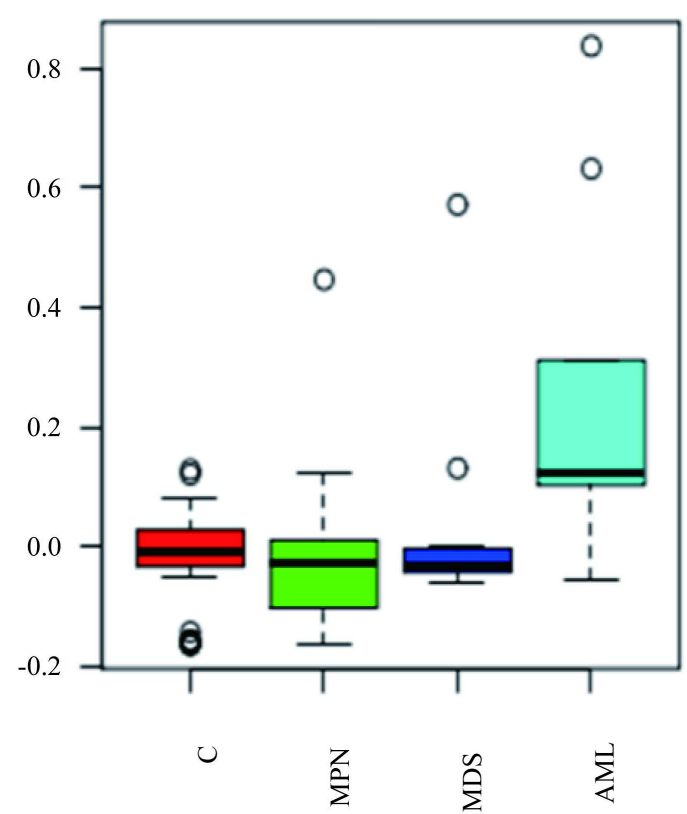

Figure 5. Total EE is detected at different concentrations in the control groups, MPN, MDS and AML. pValue $=4.5211 \mathrm{E}-9$ $-\log 10(\mathrm{p})=8.3448 ;$ FDR = 2.9839E-7; AML-Cont; AML-MPN; AML-MDS (ANOVA and Post-Hoc). C = Controls $(n=90)$; MPN = Myeloproliferative neoplasms $(n=43)$; MDS = Myelodysplastic syndromes $(n=11)$; AML = Acute myeloid leukemia $(n=9)$. 
Total MAG

Original Conc.

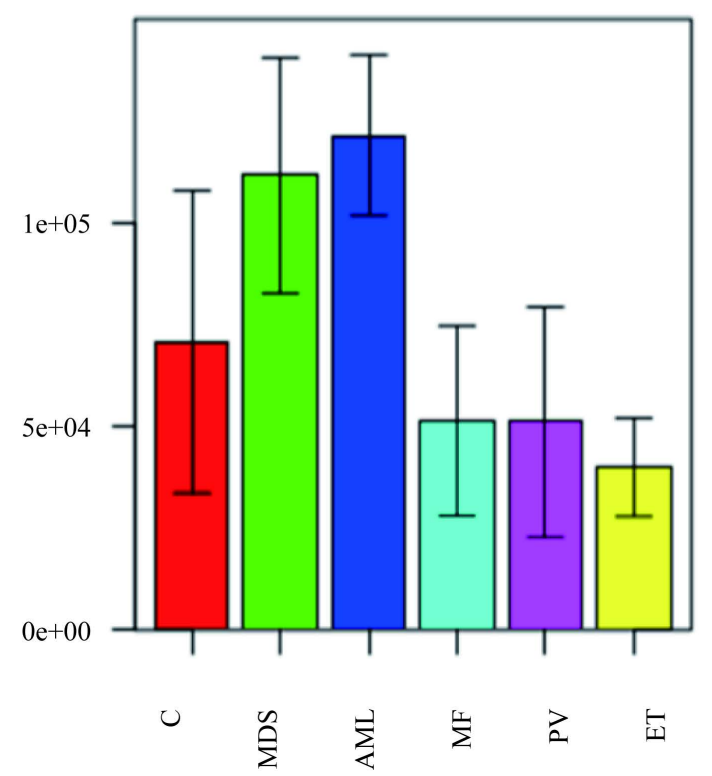

Normalized Conc.

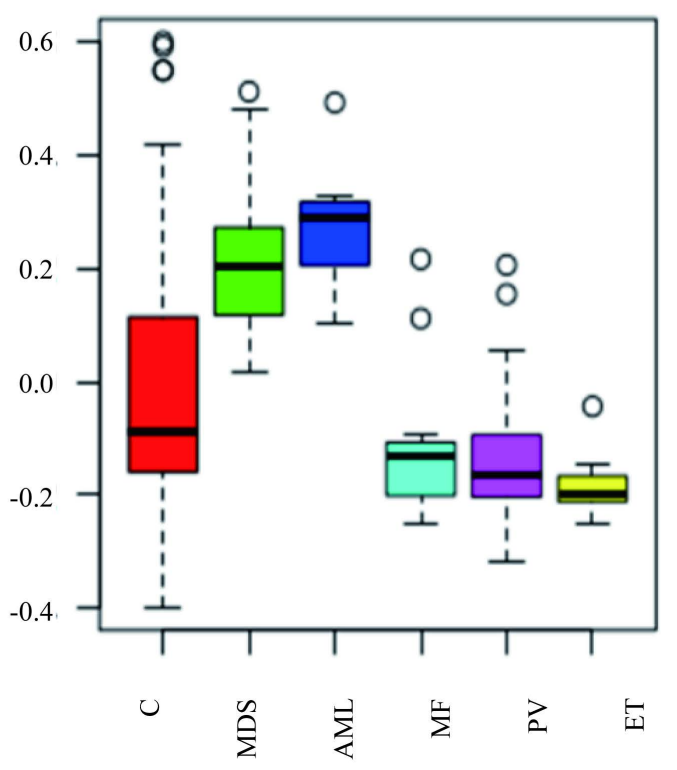

Figure 6. Total MAG is detected at different concentrations them Control groups, MPN (MF, PV and ET), MDS and AML pValue $=1.1907 \mathrm{E}-8-\log 10(\mathrm{p})=7.9242 ; \mathrm{FDR}=1.8009 \mathrm{E}-7 ;$ AML-C; C-MF; C-PV; MDS-C; C-ET; AML-MF; AML_PV; AML_ET; MDS-MF; MDS_PV; MDS-ET (ANOVA and Post-Hoc). C = Controls ( $=90)$; MPN = Myeloproliferative neoplasms $(n=43)$; MDS = Myelodysplastic syndromes $(n=11)$; AML = Acute myeloid leukemia $(n=9)$.

\subsection{Identification of Potential Biomarkers}

ROC Curve Analysis revealed Total LMMPE as the strongest discriminating marker between Controls from Patients with MDS or AML (Sensitivity = 0.95 (0.824 - 1); Specificity = 0.8941 (0.847 - 0953); Positive Likelihood Ratio $=8.972$ and Negative Likelihood Ratio $=0.05592$ and T Test $=7.576 \mathrm{E}-12$ (Figure 7(a)). In addition, these lipids were also able to differentiate MDS and AML from MPN (Sensitivity $=0.9118(0.824-1)$, Specificity $=0.95(0.85-1)$, Positive Likelihood Ratio $=18.2$ and Negative Likelihood Ratio $=0.05592)($ Figure $7(b))$.

\section{Discussion}

Up to the moment, it was not found studies of Lipidomics and/or Metabolomics using blood plasma of Myeloid Neoplams. Thus, there was the innovative idea of assessing metabolomic lipidic profile using Mass Spectrometry for those types of Neoplasms.

Among lipidic classes belonging to lipdomics, it was analysed metabolites belonging to lipids categories constituting biologic membrane, namely, Glycerophospholipids, Sphingolipids and Sterols.

Glycerophospholipids are omnipresent in the nature. They are the majority in composition of cell membranes and act as a construction structure for extra and intracellular proteins. They are involved in cell proliferation and apoptosis. In favor of Lipidomic research worldwide, Fahy and collaborators standardized the nomenclature of glycerophospholipids class as phospholipid in last update in 2009.

Glycerophospholipids can be produced from a phosphatidic acid or a diacyl-glycerol. Such category can be sub divided in different classes, as a result of nature of main polar link group. Examples of glycerophospholipids found in biological membranes are phosphatidylcholines (PC), phosphatidylethanolamines (PE), phosphatidylinositol (PI) and phosphatidylserine (PS) (LIPID MAPS). We have found the phospholipids in this work: MAG, GlcdE, LPA, SQDG, ME, MG, LPC, DMPE, MMPE, GP, LMMPE, PA, PC, ERG, PS, DAG.

The sphingolipids are a complex family of compounds that share a common structural characteristic, a structure of esfingoid basis, which is synthesized again from serine, and a fatty acid of long chain of acyl-CoA, subsequently converted into ceramides, phosphosphingolipids, glycosphingolipids, glycosphingolipids and other 


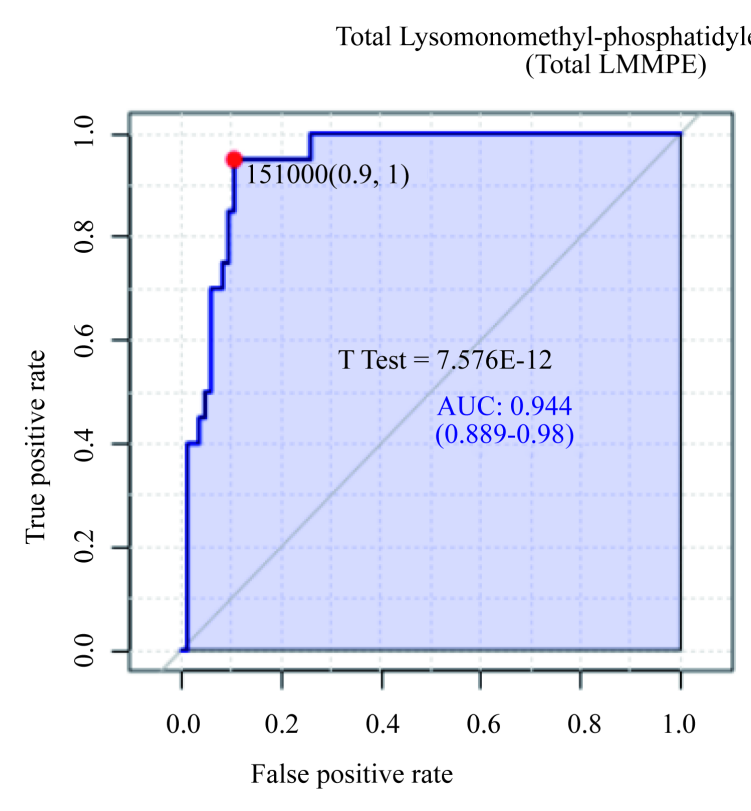

(a)

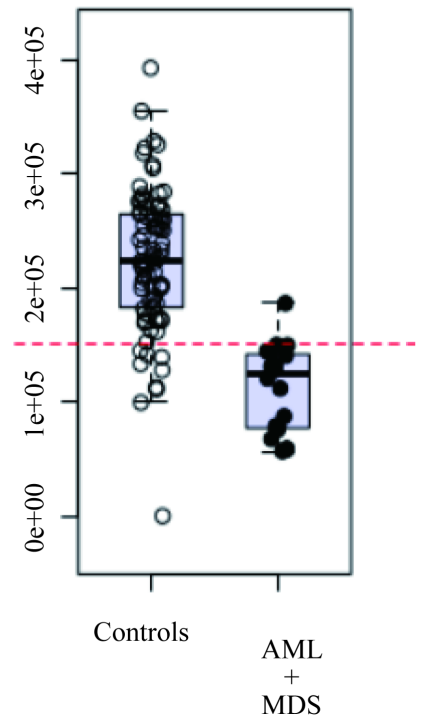

Total Lysomonomethyl-phosphatidylethanolamine (Total LMMPE)
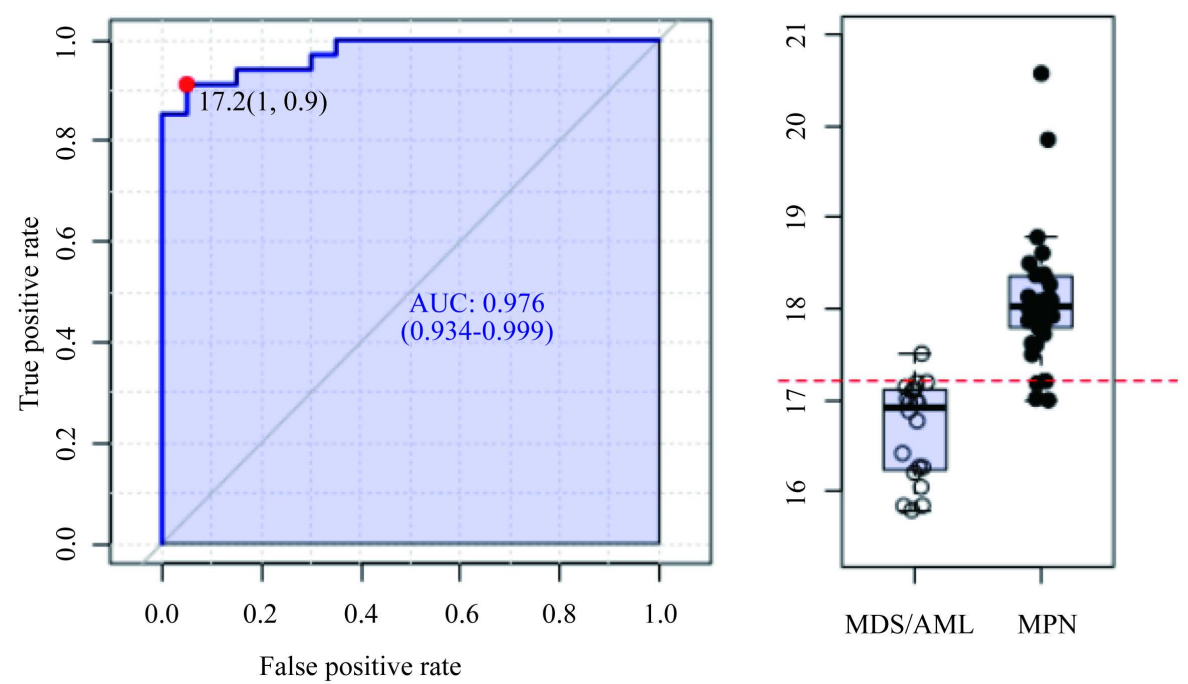

(b)

Figure 7. ROC curve analysis total LMMPE. (a) Discriminating marker between Controls from Patients with MDS or AML (Sensitivity $=0.95(0.824-1)$; Specificity $=0.8941$ (0.847 0953); Positive Likelihood Ratio $=8.972$ and Negative Likelihood Ratio $=0.05592$ and $\mathrm{T}$ Test = 7.576E-12); (b) Differentiate MDS and AML from MPN (Sensitivity $=0.9118$ (0.824 - 1), Specificity = 0.95 (0.85 - 1), Positive Likelihood Ratio = 18.2 and Negative Likelihood Ratio $=0.05592)$.

species, including proteins. The main basis of sphingoid of mammals is commonly referred to as sphingosine. The latter are found in a number of compounds, including liso sphingolipids (LIPID MAPS).

The ceramides, considered the simplest lipid among the sphingolipids consist of an esfingoid basis and a fatty acid connected to $\mathrm{C}-2$ via $\mathrm{N}$-acylation. They usually contain fatty acids, having variation in length of chain and degree of hydroxylation, although monounsaturated fatty acids, particularly with a very long chain, are also 
found among sphingolipids [13]. The main phosphosphingolipids of mammals are sphingomyelins.

The deregulated metabolism of sphingolipids became a common point among human cancers. Those lipids are part of an essential class for cellular structural integrity and perform a function of regulating fluidity of lipidic bilayer. The sphingolipids metabolites also act as messengers of bioactive lipids and contribute in intracellular signalization through activation or inhibition of receptors, kinases and phosphatase proteins and transporters of ions. They are known for influencing cellular functions, such as growth, differentiation and apoptosis [14]. We found at this work the metabolites GCer E and CerP, belonging to sphingolipids category.

The Metabolomics provides a dynamic portrait of metabolic condition of an organism, in other words, it accessed the final product of genetic expression and influences of a certain physical-pathological condition [15]. The studies in this field can be divided according to two main objectives: those where the interest is understanding biological processes from lipidic profiles found in health and disease and those that have as target the development of biomarkers [12].

\section{Conclusions}

Using plasma to identify new biomarkers is relevant, as it is more accessible and its obtainment is less intrusive, compared with other tissues. Its composition seems to be altered in any disturbance of homeostasis of blood cells.

It was observed in this work a prospective biomarker discriminating of studied phenotypes. Although we have used an undirected analysis—- "untargeted"-we considered the results as promising. Future studies starting from directional analysis can complement those findings and assess better LMMPE performance.

\section{Competing Interests}

The authors declare that they have no competing interests.

\section{Authors Contributions}

ARO carried out samples obtainment, lipids extraction and drafted the manuscript. IDCGS participated in the design of the study and performed statistical analysis. EGLT participated in sample preparation, HAMJ participated in carried out mass spectrometry analysis and MLLFC conceived of the study, and participated in its design and coordination and helped to draft the manuscript. All authors read and approved the final manuscript.

\section{Acknowledgements}

We thank our colleague Maysa Seabra Cendoroglo from Federal University of São Paulo, Department of Medicine, Division of Geriatrics and Gerontology and Blood Center of Sao Paulo Hospital who provided greatly assisted to sample obtention. The fellowship grant supported by CNPq was Adriana Ramos de Oliveira, process number 134155/2013-0.

\section{References}

[1] Vardiman, J.W., Thiele, J., Arber, D.A., Brunning, R.D., Borowitz, M.J., Porwit, A., Harris, N.L., Le Beau, M.M., Hellström-Lindberg, E., Tefferi, A. and Bloomfield, C.D. (2009) The 2008 Revision of the World Health Organization (WHO) Classification of Myeloid Neoplasms and Acute Leukemia: Rationale and Important Changes. Blood, 114, 937951. http://dx.doi.org/10.1182/blood-2009-03-209262

[2] National Cancer Institute (2013) National Cancer Institute SEER Cancer Statistics Review 1975-2010. National Cancer Institute, 1992-2010.

[3] Dennis, E.A. (2009) Lipidomics Joins the Omics Evolution. Proceedings of the National Academy of Sciences of the United States of America, 106, 2089-2090. http://dx.doi.org/10.1073/pnas.0812636106

[4] Han, X. and Gross, R.W. (2005) Shotgun Lipidomics: Electrospray Ionization Mass Spectrometric Analysis and Quantitation of Cellular Lipidomes Directly from Crude Extracts of Biological Samples. Mass Spectrometry Reviews, 24, 367-412. http://dx.doi.org/10.1002/mas.20023

[5] Wenk, M.R. (2010) Lipidomics: New Tools and Applications. Cell, 143, 888-895. http://dx.doi.org/10.1016/j.cell.2010.11.033

[6] Roberts, L.D., McCombie, G., Titman, C.M. and Griffin, J.L. (2008) A Matter of Fat: An Introduction to Lipidomic 
Profiling Methods. Journal of Chromatography B, 871, 174-181. http://dx.doi.org/10.1016/j.jchromb.2008.04.002

[7] Wymann, M.P. and Schneiter, R. (2008) Lipid Signalling in Disease. Nature Reviews Molecular Cell Biology, 9, 162176. http://dx.doi.org/10.1038/nrm2335

[8] Murphy, R.C., Raetz, C.R.H., Reynolds, C.M. and Barkley, R.M. (2005) Mass Spectrometry Advances in Lipidomica: Collision-Induced Decomposition of $\mathrm{Kdo}_{2}$-Lipid A. Prostaglandins \& Other Lipid Mediators, 77, 131-140. http://dx.doi.org/10.1016/j.prostaglandins.2004.09.004

[9] Gross, R.W. and Han, X. (2011) Lipidomics at the Interface of Structure and Function in Systems Biology. Chemistry \& Biology, 18, 284-291. http://dx.doi.org/10.1016/j.chembiol.2011.01.014

[10] Karpova, M.A., Moshkovskii, S.A., Toropygin, I.Y. and Archakov, A.I. (2010) Cancer-Specific MALDI-TOF Profiles of Blood Serum and Plasma: Biological Meaning and Perspectives. Journal of Proteomics, 73, 537-551. http://dx.doi.org/10.1016/j.jprot.2009.09.011

[11] Rübsaamen, K. (2010) Lipidomic Analysis of Circulating Human Blood Cells. Doctoral Thesis, University of Regensburg, Regensburg. http://epub.uni-regensburg.de/13246/1/Dissertation_Katharina_Ruebsaamen.pdf

[12] Xia, J., Broadhurst, D.I., Wilson, M. and Wishart, D.S. (2013) Translational Biomarker Discovery in Clinical Metabolomics: An Introductory Tutorial. Metabolomics, 9, 280-299. http://dx.doi.org/10.1007/s11306-012-0482-9

[13] Lahiri, S. and Futerman, A.H. (2007) The Metabolism and Function of Sphingolipids and Glycosphingolipids. Cellular and Molecular Life Sciences, 64, 2270-2284. http://dx.doi.org/10.1007/s00018-007-7076-0

[14] Ryland, L.K., Fox, T.E., Liu, X., Loughran, T.P. and Kester, M. (2011) Dysregulation of Sphingolipid Metabolism in Cancer. Cancer Biology \& Therapy, 11, 138-149. http://dx.doi.org/10.4161/cbt.11.2.14624

[15] Lv, W. and Yang, T. (2012) Identification of Possible Biomarkers for Breast Cancer from Free Fatty Acid Profiles Determined by GC-MS and Multivariate Statistical Analysis. Clinical Biochemistry, 45, 127-133. http://dx.doi.org/10.1016/j.clinbiochem.2011.10.011 